\title{
Benefícios decorrentes de prática do cuidado farmacêutico em hipertensão e diabetes tipo 2 para sua efetivação em unidades de saúde
}

\author{
Benefits from practices of pharmaceutical care in hypertension and \\ type 2 diabetes to be conducted in health units
}

Recebido em: 07/12/2016 Aceito em: $26 / 07 / 2017$

Maria Aparecida NICOLETTI; Lina Terumi KUBOTA

Faculdade de Ciências Farmacêuticas, Universidade de São Paulo. Avenida Professor Lineu Prestes, 580, CEP 05508-900.

São Paulo,SP,Brasil.E-mail:nicoletti@usp.br

\begin{abstract}
Patients with chronic diseases, especially hypertension and diabetes, need constant monitoring by health professionals, since the lack of control of both, blood glucose and blood pressure, are the precursors of fatal attacks on the kidney functions, cardiovascular problems on top of generating costs to the Public Health System. The main goal of the work is to seek evidence to corroborate the benefits of Pharmaceutical Care to patients with chronic non-communicable diseases, in particular, hypertension and diabetes. The research is a narrative review based on articles available in the scientific databases Web of Science, SciELO, Virtual Health Library, USP Bibliographic Database (DEDALUS) and PubMed-NCBI, as well as, on epidemiological data found in DATASUS as well as on Brazilian and international guidelines associated with hypertension and diabetes. The inclusion criteria established was to consider complete articles addressing the main goal of the research, from 2000 up to 2016, written in Portuguese, English or Spanish, presenting conclusive results. The exclusion criteria were satisfied by publications that did not meet the inclusion criteria. It was observed that the practice of pharmaceutical care had an impact on the adherence rate to treatment in hypertensive and/or diabetic patients, providing clinical improvement and increasing the quality of life, also generating socioeconomic benefits. It is important to emphasize that Pharmaceutical Care is not offered in isolation from other health services, but in collaboration with patients as well as with the multidisciplinary health team; by having each professional acting in their field of expertise benefits both, i.e., the patient and the public health system.
\end{abstract}

Keywords: pharmaceutical care; hypertension; type 2 diabetes mellitus; treatment adherence.

\section{RESUMO}

Pacientes portadores de doenças crônicas, em especial, hipertensão e diabetes, necessitam acompanhamento constante do profissional da saúde, pois o descontrole tanto da glicemia como da pressão arterial é precursora de crises fatais do funcionamento de rins, problemas cardiovasculares e motores, gerando custos evitáveis ao sistema público de saúde. O objetivo desse trabalho é buscar evidências que demonstrem os benefícios dos cuidados farmacêuticos a pacientes portadores de doenças crônicas não transmissíveis, em especial, hipertensão e diabetes tipo 2, por meio A pesquisa é uma revisão narrativa utilizando busca de artigos nas bases científicas de dados Web of Science, SciELO, Biblioteca Virtual em Saúde, Bancos de Dados Bibliográficos da USP (DEDALUS) e PubMed-NCBI, bem como dados epidemiológicos do DATASUS e diretrizes brasileiras, para a contextualização de hipertensão e diabetes. Foi observado que a prática de cuidados farmacêuticos impactou no índice de adesão ao tratamento em pacientes hipertensos e/ou diabéticos, proporcionando melhora clínica e na qualidade de vida gerando, ainda, um benefício socioeconômico. É importante enfatizar que o cuidado farmacêutico não é praticado isoladamente de outros serviços de saúde, mas em colaboração com os pacientes e a equipe multidisciplinar em saúde; cada profissional deve atuar em seu âmbito de competência, o que resultará em benefício ao paciente e ao setor público.

Palavras-chave: cuidado farmacêutico; hipertensão; diabetes mellitus tipo 2; adesão ao tratamento. 
INTRODUÇÃO

Durante muito tempo, o farmacêutico teve seu papel como profissional de saúde negligenciado em relação ao cuidado em saúde. As mudanças históricas nos processos produtivos e as influências decorrentes nos currículos acadêmicos culminaram em um profissional tecnicista, de conhecimentos multicompartimentados, descontextualizado da equipe multidisciplinar, mantendo relação mais íntima com o medicamento do que com o paciente. Neste sentido, é importante salientar que a atuação clínica promove o reencontro entre o farmacêutico e o paciente, exigindo do profissional novas competências para que possa novamente se responsabilizar pelo bem-estar do indivíduo e tornar-se um dos provedores de cuidados em saúde, no contexto do cuidado multidisciplinar (1).

Em 1993, a World Health Organization (WHO) reconheceu o papel fundamental do farmacêutico no sistema de atenção à saúde e, em 1994, o Europharm Forum, entidade de cooperação entre a WHO e as diversas organizações nacionais profissionais, fundou a Pharmaceutical Care Network Europe (PCNE) com o propósito de implantar e desenvolver a prática do cuidado farmacêutico na Europa, por intermédio da realização de projetos multicêntricos (2-4). No Congresso da Federação Internacional Farmacêutica (FIP), realizado em Jerusalém, foram debatidas as diretrizes para a preparação do profissional farmacêutico do terceiro milênio (5). Para tanto, a FIP decidiu se empenhar em difundir o cuidado farmacêutico, em especial nos países de terceiro mundo. Asrecomendações internacionais identificaram o cuidado farmacêutico como filosofia de prática e estabeleceram a missão da prática farmacêutica para prover medicamentos, outros serviços e produtos de saúde, bem como ajudar a sociedade a fazer o melhor uso racional e correto deles (6).

Em 2008, o Diretório Europeu da Qualidade dos Medicamentos e Cuidados de Saúde (EDQM), iniciou um estudo referente a conceitos-chave empregados e a análise dos indicadores de desempenho utilizados para avaliar a qualidade da assistência farmacêutica europeia, baseando-se na filosofia de cuidados farmacêuticos com o envolvimento do paciente com sua terapia, o monitoramento (documentação) e a cooperação multidisciplinar entre os profissionais de saúde, dentro do processo de medicação que fundamentou a publicação da Resolução ResAP-93, tratados não internacionais de cooperação dentro da União Europeia (EU). As Resoluções consideradas ResAP Partial Agreements permitem que os Estados membros se abstenham de participar de acordos dentro da Organização da EU, que dizem respeito ao papel e à formação dos farmacêuticos. Esse foi um marco importante para aprática do cuidado farmacêutico, visto que auxiliou na definição do escopo do profissional, além de definir os indicadores e os parâmetros essenciais para sua implementação em nível internacional (7).
Os serviços farmacêuticos constituem parte dos serviços de saúde e compreendem um conjunto de atividades organizadas em um processo de trabalho, que visa contribuir para a prevenção de doenças, a promoção, a proteção e a recuperação da saúde, e para a melhoria da qualidade de vida das pessoas. Os serviços farmacêuticos estão relacionados com a Farmácia Clínica, diretamente destinados ao paciente, à família e à comunidade, fundamentados pelo modelo de prática denominado "cuidado farmacêutico". Esses serviços podem ser realizados em diferentes lugares de prática, incluindo a farmácia comunitária, o leito hospitalar, a farmácia hospitalar, os serviços de urgência e emergência, os serviços de atenção primária à saúde, o ambulatório, o domicílio do paciente, as instituições de longa permanência, entre outros, segundo regulamentação específica. $\mathrm{O}$ cuidado farmacêutico é o modelo de prática que orienta a provisão de diferentes serviços farmacêuticos direcionados ao paciente, à família e à comunidade. A opção pela denominação de "cuidado farmacêutico" para a descrição deste modelo de prática deveu-se à similaridade com as outras profissões da saúde (cuidados de enfermagem, cuidado fisioterápico e cuidado nutricional), bem como ao significado da própria palavra $(8,9)$.

O cuidado farmacêutico é a prática da farmacoterapia com o propósito de melhorar a qualidade de vida e promover saúde ao paciente. É fruto do engajamento do profissional farmacêutico, do paciente e da multidisciplinaridade entre os profissionais da saúde de diversas áreas que juntos, planejam, monitoram a terapia e influenciam significantemente na mudança de comportamento do paciente. Dessa maneira, tem como objetivo conseguir o melhor método de tratamento, a prevenção de doenças e a minimização de erros terapêuticos para a segurança do paciente e a efetividade do medicamento (10).

A expansão das atividades clínicas do farmacêutico ocorreu, em parte, como resposta ao fenômeno da transição demográfica e epidemiológica observado na sociedade. A crescente morbimortalidade relativa às doenças decorrentes dos agravos não transmissíveis e da farmacoterapia repercutiu nos Sistemas de Saúde e exigiu novo perfil do farmacêutico. Nesse contexto, o farmacêutico contemporâneo atua no cuidado direto ao paciente, promove o uso racional de medicamentos e de outras tecnologias em saúde, redefinindo sua prática a partir das necessidades dos pacientes, família, cuidadores e sociedade (11).

Deve ser salientada a importância da publicação da Lei 13.021, de 08 de agosto de 2014, que estabelece a assistência farmacêutica como sendo o conjunto de ações e de serviços que visa assegurar a assistência terapêutica integral e a promoção, a proteção e a recuperação da saúde nos estabelecimentos públicos e privados que desempenhem atividades farmacêuticas, tendo o medicamento como insumo essencial e visando ao seu acesso e ao seu uso racional; e a farmácia como sendo uma 
unidade de prestação de serviços destinada a prestar assistência farmacêutica, assistência à saúde e orientação sanitária individual e coletiva (12).

Diferentemente de outros serviços prestados pelo farmacêutico, o serviço de cuidado coloca o paciente como foco principal da prática profissional e não o medicamento. Ainda que a preocupação com os problemas relacionados à farmacoterapia seja um ponto chave da atuação clínica, o foco principal deve ser a saúde e o bem-estar do paciente de maneira global, por isso é necessário compreender o outro para que se possa criar uma relação terapêutica (1).

Um estudo publicado em 2001, nos Estados Unidos da América do Norte (EUA), estimou que quando os farmacêuticos participaram ativamente da revisão das prescrições e da orientação aos idosos quanto ao uso da farmacoterapia, ocorreu diminuição dos gastos do sistema de atenção à saúde (ambulatórios e hospitais) com a redução de problemas relacionados aos medicamentos, da ordem de US\$ 3,6 bilhões por ano (13).

Em concordância aos benefícios decorrentes da atuação do farmacêutico, estudo desenvolvido por Strand e cols (2004) avaliou o impacto econômico do serviço de cuidado farmacêutico no período de janeiro de 2000 a dezembro de 2003. Foram avaliados 2.985 indivíduos, sendo que $61 \%$ deles possuíam, pelo menos, algum problema relacionado ao medicamento, e $83 \%$ mantiveram a enfermidade sob controle. Devido à atuação do farmacêutico, nessa análise foi estimada a redução de US\$ 1.134.162,00 nos custos do tratamento (14). Esses dados que estimulam investigações farmacoeconômicas, podem ser espelladas para a realidade brasileira, pois a implementação de programas de cuidado farmacêutico, atendendo grupos maiores de pacientes, poderia minimizar gastos desnecessários com internações ou complicações provocadas pela incidência de problemas medicamentosos, redirecionando os recursos públicos para a melhora da estrutura e dos serviços de saúde, incluindo a expansão do acesso aos medicamentos (15).

As doenças crônicas não transmissíveis (DCNT) vêm se destacando como importante desafio de saúde pública há muitos anos, principalmente, pela morbidade e mortalidade que causam. Estas doenças podem provocar sérios graus de incapacidade que afetam tanto os hábitos de vida e o bem-estar do indivíduo quanto à economia do país (16). São multifatoriais e de longa duração e se desenvolvem no transcorrer da vida sendo consideradas como problema de Saúde Pública. No Brasil, em 2013, as DCNT foram a causa de aproximadamente $72,6 \%$ das mortes $(17,18)$ e, segundo a World Health Organization, em 2008, foram responsáveis por $63 \%$ das mortes no mundo (19).

Segundo Maltta e cols (2015) dois agravos à saúde merecem especial atenção, a hipertensão e o diabetes tipo 2 (DM2), em razão de atingirem todas as camadas socioeconômicas, além de se encontrarem entre os mais prevalentes na população de 18 anos ou mais de idade, sendo classificados, respectivamente, em $1^{\circ}$ e $5^{\circ}$ lugares. Em números absolutos, há aproximadamente 31 milhões de indivíduos acometidos com hipertensão e acima de 9 milhões de diabéticos. Em relação ao diabetes, os autores, também, citam nessa publicação que, segundo a WHO, em 2008, este agravo à saúde foi responsável por 1,3 milhão de mortes e cerca de $4 \%$ das mortes prematuras, além de estar associado com limitações e incapacidade dos indivíduos (20).

Portanto, o cenário mostra a necessidade de ações de prevenção da doença e promoção da saúde, as quais poderão ser realizadas por meio da institucionalização da prática do cuidado farmacêutico em suas diferentes vertentes.

O objetivo do desenvolvimento do texto é evidenciar o benefício decorrente da prática do cuidado farmacêutico em doenças crônicas não transmissíveis, em especial, a hipertensão e o diabetes tipo 2, para sua efetivação aos pacientes nas Unidades de Saúde.

\section{MÉTODOS}

A revisão bibliográfica narrativa foi realizada utilizando como descritores em português: Atenção Farmacêutica, diabetes, hipertensão, farmacoterapia, qualidade de vida, continuidade da Assistência ao Paciente, Pacientes Desistentes do Tratamento, custos farmacológicos hipertensão e diabetes; em espanhol: Atención Farmacéutica, Continuidad de la Atención al Paciente, Cualidad de Vida, Pacientes Desistentes del Tratamiento; e em inglês Pharmaceutical Care, Treatment Effectiveness, Pharmaceutical Management, Pharmaceutical Policy, Pharmacotherapy, Patient Dropouts, Continuity of Patient Care, Quality of Life, Diabetes and Pharmacentical Care, Hypertension and Pharmaceutical Care, and Pharmaceutical Care nas seguintes bases de dados Web of Science, Scientific Eletronic Library Online, Biblioteca Virtual em Saúde, Banco de Dados Bibliográficos da USP (DEDALUS) e U.S. National Library of Medicine - National Institute of Health.

Os critérios de inclusão estabelecidos foram artigos completos e que abordassem o objetivo do texto, no período de tempo proposto, em língua portuguesa, inglesa e espanhola com resultados conclusivos. Os critérios de exclusão referiam-se a artigos que objetivamente não tratassem sobre o tema abordado, publicados fora do período proposto, em língua estrangeira que não fosse inglês ou espanhol, além de resultados não conclusivos.

A busca abrangeu o período de 2000 a 2016, considerando que ao final dos anos 90 houve a publicação da Política Nacional de Medicamentos cujo impacto começou a ser sentido a partir de 2000.

Também foram levantados dados epidemiológicos a partir do DATASUS e de diretrizes brasileiras e inter- 
nacionais para a contextualização dos agravos à saúde, hipertensão e diabetes, para melhor entendimento do impacto para população.

\section{CONTEXTUALIZAÇÃO DO AGRAVO À SAÚDE: HIPERTENSÃO}

Epidemiologia e Desafios da Hipertensão. No Brasil, com base no levantamento do DATASUS realizado em 2016, no período de 5 anos, o número de hipertensos registrados foi de 1.473.254, sendo que 506.114 são portadores de hipertensão e diabetes (21).

Segundo as Diretrizes Brasileiras de Hipertensão, a hipertensão arterial sistêmica (HAS) é condição clínica multifatorial caracterizada pelo alto nível e sustentada da pressão arterial (PA). Pode estar associada, frequentemente, a alterações funcionais e/ou estruturais dos órgãos-alvo (coração, encéfalo, rins e vasos sanguíneos) e aos distúrbios metabólicos que, como consequência, aumentam o risco de eventos cardiovasculares, o desenvolvimento de acidente vascular cerebral, a insuficiência renal, a morte prematura e a invalidez (22).

Considerando que a hipertensão é doença silenciosa, mas sintomática quando em estágio avançado, a intensificação da comunicação e a informação para a população sobre sua incidência é um meio efetivo para a sua detecção precoce. Assim, o aumento da cobertura de assistência e a promoção à saúde pública para uma abrangência universal tornam-se fundamentais para sustentar a manutenção de uma vida saudável. O controle da hipertensão não é fácil e exige comprometimento entre o paciente e o profissional da saúde. O sucesso no controle e tratamento está fundamentado, principalmente, no acesso a medicamentos hipertensivos de qualidade, nos cuidados primários do atendimento à saúde, na adesão dos pacientes às ações que assegurem o seu controle e a participação em programas sociais para hábitos saudáveis de vida. A hipertensão, por ser considerada uma das DCNT, desafia gestores públicos para o seu controle e tratamento, além de constituir razão de gasto e perdas econômicas grandiosas (17).

Nem toda população diagnosticada com hipertensão e em uso de anti-hipertensivos é não-responsiva ao tratamento ou capaz de aderir ao tratamento correto. A baixa adesão farmacológica de pacientes hipertensivos é devida a 3 fatores principais, a saber, a situação socioeconômica, a assistência ao serviço de saúde características pessoais, além outros relacionados ao tratamento farmacológico (23).

Tratar as complicações da hipertensão não controlada implica em intervenções dispendiosas, pois traz como consequências, a cirurgia cardíaca, a diálise, os leitos ocupados por hospitalização, que elevam significantemente os orçamentos individuais e governamentais para manutenção da saúde desses pacientes, além de óbitos que poderiam ser evitados. De acordo com dados de 2013, da WHO, a hipertensão é responsável por, ao menos, $45 \%$ das mortes causadas por doenças cardíacas e $51 \%$ das causas de mortes por acidentes vasculares. As projeções epidemiológicas estimaram que, em 2025, $75 \%$ da população em países emergentes (ou 1,17 bilhão de pessoas) serão hipertensas (24).

Doenças no aparelho circulatório foram responsáveis por mais de 800 mil internações processadas pelo Sistema Único de Saúde (SUS) em 2006, com um custo aproximado de R\$1,3 bilhão, e se mantém como principal causa agrupada de mortes no Brasil. A Região Sul apresenta a maior taxa de internações por doenças cardiovasculares (86 por 10 mil habitantes em 2006), com os custos médios de internação mais elevados, e as maiores taxas brutas de mortalidade do País (23).

A prevenção e o controle da hipertensão arterial são complexos, o que exige a colaboração de múltiplas partes interessadas, incluindo os governos, os profissionais da saúde, a sociedade civil, as universidades e a indústria de alimentos e bebidas considerando que, tanto os tratamentos farmacológicos, como os não farmacológicos, impactam sobremaneira na qualidade de vida e na segurança do indivíduo. Saliente-se, também, a importância da educação em saúde para a participação ativa do indivíduo na colaboração das ações necessárias para o controle deste agravo à saúde.

Hipertensão relacionada a questões socioeconômicas. Lessa (2006) afirmou que o impacto da hipertensão arterial sistêmica descontrolada é determinado pelo número de casos de acidente vascular cerebral (AVC), doenças isquêmicas do coração, insuficiência cardíaca, insuficiência renal e isquemia vascular periférica (25). Como consequências, o setor da saúde contabiliza diversos recursos dispensados, os quais se destacam: elevados custos hospitalares dos eventos agudos e da própria HAS não controlada, além dos efeitos do tratamento, com ou sem adesão, que são detectados em longo prazo por estatísticas de tendências viabilizadas pelo monitoramento dos fatores de risco comportamentais e por inquéritos periódicos de saúde. Efeitos negativos sobre a hipertensão decorrem, não somente da não adesão ao tratamento como, também, do sub tratamento, inadequação do medicamento, dificuldade no acesso ao sistema de saúde, indisponibilidade de medicação na Rede Básica de Saúde, quantidade de medicamento, número de doses diárias da medicação prescrita, efeitos adversos, resistência ao tratamento e presença de comorbidades (25).

A grande preocupação com a mortalidade por acidente vascular cerebral, atribuída ao descontrole da hipertensão arterial sistêmica, deve-se ao fato dos pacientes estarem em idade economicamente produtiva. O impacto social negativo é percebido pelas elevadas proporções de casos nesta faixa etária (24). 
Segundo Lessa (2006) dos 20 aos 64 anos de idade (época da aposentadoria), as internações decorrentes de eventos cardiovasculares precoces somaram 415.018 (49\% do total) e os seus custos alcançaram R\$ 645.963.613,40 (33,5\% do total) (25). No Brasil, no entanto, essa é uma parte importante do impacto social da não adesão ao tratamento, inadequação do tratamento ou do não tratamento, tendo em vista que a prevalência da hipertensão é muito elevada entre os 30 e 60 anos de idade e com desfechos graves. Dessa forma, o prejuízo social torna-se ainda maior, na medida em que os gastos que ocorrem são elevados, mas não permitem o alcance das metas dos programas contra a hipertensão (25).

Hipertensão e o cuidado farmacêutico. Tanto o tratamento medicamentoso como o não medicamentoso da HAS dependem da progressão da doença e da classificação de risco; e o objetivo primordial é reduzir a morbidade e a mortalidade cardiovascular. Dessa forma, o tratamento não farmacológico baseia-se em mudanças de estilo de vida, como por exemplo, o controle do peso, a redução da ingestão de álcool e de sódio, de gordura saturada e de colesterol, além da ingestão adequada de sais minerais, associados a ações como cessar o tabagismo, praticar regularmente atividades físicas e controlar o estresse à medida do possível. Além disso, as práticas farmacêuticas envolvendo as consultas farmacêuticas, as intervenções e o monitoramento de registros terapêuticos, contextualizados na proposta de acompanhamento terapêutico do indivíduo provaram a efetividade no tratamento da hipertensão e na redução do risco de doenças cardiovasculares (22).

Cazarim e cols (2016) em análise quasi-experimental (pesquisa para estimar o impacto causal de uma intervenção sobre a sua população-alvo) realizada com controles retrospectivos de dados entre 2006 e 2012 concluíram que o cuidado farmacêutico realizado em duas Unidades Básicas de Saúde Pública de Ribeirão Preto, SP foi efetivo no controle da PA em 104 pacientes hipertensos (26). Pacientes mostraram redução e o controle da PAS de 11,5/5,7 mmHg após o programa. Os resultados desta análise mostraram que o cuidado farmacêutico proporcionou de $54 \%$ a $100 \%$ de adesão à farmacoterapia pelos pacientes, demostrando o papel fundamental da atuação do farmacêutico. Além disso, utilizando o instrumento de avaliação denominado 36Item Short-Form Health Survey (SF-36), houve aumento na pontuação nos oito domínios que avaliam: capacidade funcional, aspectos físicos, dor, estado geral de saúde, vitalidade, aspectos sociais, aspectos emocionais e saúde mental, ou seja, em média, um aumento de 66,8 a 85,3 pontos e melhoria de $27,6 \%$ na qualidade de vida desses pacientes (26).

Nos EUA, estudo realizado em farmácias comparou os benefícios do cuidado farmacêutico para os pacientes que frequentavam aquelas unidades. Para este comparativo, foram estabelecidas nove farmácias onde os pacientes receberiam os cuidados assim que houvesse a dispensação do medicamento. Durante 12 meses de acompanhamento destes pacientes foi observado que aqueles que o receberam apresentaram redução de PA sistólica e diastólica. Além disso, a taxa de adesão ao anti-hipertensivo se apresentou elevada e constante durante todo o período de estudo (27).

Martins e cols (2013) realizaram um estudo longitudinal de cuidado farmacêutico em pacientes com Hipertensão Arterial assistidos pelo Programa Estratégia Saúde da Família (ESF). Foram registrados 142 Problemas Relacionados a Medicamentos (PRM) sendo o mais frequente a falta de efetividade do tratamento $(33,8 \%)$, seguido pelos problemas de indicação $(24,6 \%)$, segurança do tratamento $(23,2 \%)$ e adesão $(18,3 \%)$. Em relação às intervenções farmacêuticas, os autores descreveram o total de 135 , das quais $92,6 \%$ foram realizadas com a comunicação farmacêutico-paciente, e que $48,8 \%$ foram implementadas. $\mathrm{O}$ modelo de cuidado farmacêutico foi capaz de detectar PRM, propondo intervenções para resolvê-los e preveni-los e, consequentemente, contribuindo para a melhora de parâmetros clínicos, como a glicemia de jejum e o risco cardiovascular (28).

O trabalho em equipe multiprofissional é fundamental no sentido de garantir cuidados de saúde integrados no Programa Estratégia de Saúde da Família, uma vez que permite diferentes formas de lidar com o paciente e a sua família dentro do processo de intervenção, facilitar o intercâmbio de informações entre profissionais da equipe com o objetivo de otimizar resultados e estabelecer melhor interação entre a equipe e a comunidade (29).

Outro ponto a ser considerado para a inclusão e a extensão do cuidado farmacêutico nos Serviços de Saúde é a melhora da qualidade de vida dos pacientes. Um estudo realizado na Nigéria com 65 pacientes utilizou o questionário denominado Short Form Health Survey $\left(\mathrm{SF} 12^{\circledR}\right)$ como métrica para avaliar o impacto do cuidado na qualidade de vida dos pacientes. Os profissionais foram fundamentais para a detecção de todos problemas relacionados ao medicamento e a prática do cuidado melhorou significativamente a qualidade de vida nos quesitos: melhoria da capacidade mental e de componentes de dor (30).

Vista sob perspectiva crítica, a deficiência da adesão, entre os portadores de hipertensão arterial, tem relação direta com os diversos fatores associados à falta de informação sobre o tratamento e a doença. A educação do paciente pode proporcionar a conscientização quanto ao seu estado de saúde e à necessidade do uso correto dos medicamentos, tornando o tratamento mais efetivo e seguro, além de levar a maior interação entre os profissionais de saúde (em especial o médico, o farmacêutico e o enfermeiro), podendo reduzir diversos problemas medicamentosos, da prescrição à administração, colaborando na redução de custos do sistema de saúde (31). 
A elevada incidência de morbimortalidade relacionada a medicamentos pode ser prevenida ou amenizada por meio da reorganização da forma como os medicamentos são gerenciados pelos serviços de saúde, os quais devem apresentar como foco principal a Assistência Farmacêutica (em sua contextualização em termos de política de medicamentos) e a equipe de saúde voltada para a gestão clínica da farmacoterapia e dos desfechos em saúde, com o objetivo do uso racional de medicamentos (32).

De maneira geral, pode ser considerado que o cuidado farmacêutico se encontra, ainda, incipiente no Brasil, tanto no setor público quanto no privado, apesar de algumas iniciativas já estarem sendo desenvolvidas. Considerando o setor público, na visão administrativo-financeira, os gestores precisam estar conscientizados dos benefícios de sua implantação e a sua relação direta com a redução dos custos decorrentes, entretanto, o principal beneficiário é o usuário SUS.

\section{CONTEXTUALIZAÇÃO DO AGRAVO À SAÚDE: DIABETES TIPO 2}

Epidemiologia. Nos últimos trinta anos, a prevalência de diabetes tipo 2 aumentou drasticamente nos países em todos os níveis desenvolvimento. Estima-se que o número de diabéticos no mundo seja aproximadamente de 387 milhões, com crescente aumento, e projeta-se 471 milhões para 2035 (33). Aproximadamente $80 \%$ desses indivíduos vivem em países em desenvolvimento, onde a presença deste agravo à saúde apresenta maior intensidade e está em elevação. Sabe-se que a proporção de pessoas acometidas em faixas etárias mais jovens, vem sendo crescente nas últimas décadas e sua magnitude está causando grande preocupação na saúde global (17).

Em relatório de 2016, a WHO identificou que a prevalência global (idade-padronizada) de diabéticos quase duplicou desde 1980 , passando de $4,7 \%$ para $8,5 \%$, na população adulta. Existem 422 milhões indivíduos diabéticos no mundo e durante a última década, a prevalência aumentou mais rapidamente em países de baixa e média renda, do que nos de alta renda. Existe forte correlação deste aumento com envelhecimento populacional, maior urbanização, aumento da prevalência da obesidade e sedentarismo. Dessa forma, conseguir quantificar o predomínio atual e estimar o número de pessoas com diabetes no futuro é essencial para o planejamento e a alocação de recursos financeiros públicos de maneira racional e igualitária à população, pois é uma das maiores causas de óbitos ou invalidez (34).

Em 2012, foram registrados 1,5 milhões de óbitos causados pelo diabetes (35). A alta taxa descontrolada de glicose no sangue resultou em um adicional de 2,2 milhões de mortes, em função do aumento dos riscos de doenças cardiovasculares e comorbidades crônicas.
Quarenta e três por cento dessas, ou seja, 3,7 milhões de mortes ocorrem antes da idade de 70 anos e é a prevalência é maior em países de baixa e média renda. Essa prevalência pode ser maior, considerando que o atestado de óbito, normalmente, não constar o diabetes tipo 2 como sendo a causa mortis, pelo fato de suas complicações serem em níveis cardiovasculares e cerebrovasculares sendo, então, essas últimas descritas como as responsáveis pelo óbito (35).

Para manter a qualidade de vida sem comprometimento da vida cotidiana, os diabéticos necessitam tratamento adequado, acesso a medicamentos hipoglicemiantes, insulina, mudanças de hábitos sedentários e alimentação adequada que são fundamentais para a sua sobrevivência, ou seja, tratamento farmacológico e não farmacológico (36).

Custos e aspectos socioeconômicos. Sabe-se que os custos médicos diretos associados ao diabetes tipo 2 incluem os gastos com a prevenção, o tratamento e suas complicações. A isso, são incluídos atendimento ambulatorial e de emergência; internação hospitalar; medicamentos e suprimentos médicos, como dispositivos de injeção e equipamentos para saúde de automonitoramento e cuidados de longa duração. Com base em estimativas de custo decorrente de revisão sistemática recente, foi projetado que o custo anual direto de diabetes mellitus para o mundo é maior do que US\$ 827 bilhões (37). A International Diabetes Federation (IDF) estimou que os gastos globais com este agravo à saúde mais do que triplicou durante o período de 2003 a 2013 (38).

Calcula-se que os gastos diretos com este agravo à saúde variam entre 2,5 e $15 \%$ do orçamento anual da Saúde de um país, e dependem de sua prevalência e do grau de complexidade do tratamento disponível (33). Para o Brasil, estimativas do custo direto oscilam em torno de US\$ 3,9 bilhões e cálculos, de 2016, das despesas com o tratamento ambulatorial por meio do SUS foram da ordem de US $\$ 2.108,00$ por paciente, dos quais US\$ $1.335,00$ estavam relacionados a custos diretos (34). Analisando o diabetes tipo 2 como diagnóstico principal, o custo annual foi de aproximadamente R\$ 40,3 milhões, sendo 91\% decorrentes de internações hospitalares devido complicações. Esse valor foi subestimado, pois é comum pacientes atendidos por outros agravos à saúde relacionados, como a obesidade, também apresentarem diabetes (33).

O diabetes não tratado pode ser incapacitante, podendo impedir o indivíduo de continuar a trabalhar ou diminuir o desempenho profissional pelas limitações crônicas como: retinopatias, neuropatias e insuficiência renal. Segundo a American Diabetes Association (2013) o custo total estimado do diabetes diagnosticado em 2012, nos EUA, foi US\$ 245 bilhões, incluindo US\$ 176 bilhões em custos médicos diretos e US\$ 69 bilhões em produtividade reduzida, sendo que o maior componente da despesa foi o cuidado hospitalar (43\% 
do custo médico total). As pessoas com o diagnóstico desse agravo à saúde, em média, têm despesas médicas aproximadamente 2,3 vezes maiores do que as despesas em sua ausência (39).

Um estudo realizado no período 2007-2011 com grupos de pacientes que receberam tratamento com e sem cuidado farmacêutico concluiu que o resultado de custos indiretos por absentismo e os custos devido a óbitos por pacientes diabéticos foi $\mathrm{R} \$ 11.781,19$, por paciente. Os custos indiretos por paciente que tinham o cuidado foram R $\$ 2.119,21$. Somando-se aos custos diretos, o custo dos pacientes que não tinham o cuidado farmacêutico durante o percurso terapêutico resultou em $R \$ 15.020,72$, enquanto que nos grupos de pacientes com o cuidado farmacêutico foi de $\mathrm{R} \$ 6.141,86$. Considerando esta análise, o serviço é a alternativa de menor custo-efetividade para o tratamento de diabetes tipo 2 . Sua razão de custo incremental para anos de vida demonstrou economia de $\mathrm{R} \$ 1.430,99$ reais por benefício extra, ou seja, efetividade no controle glicêmico, da hemoglobina glicada (HbAlc) e anos de vida ganhos (40).

É preciso esclarecer que investir no combate ao diabetes tipo 2, gera benefícios, em longo prazo, para as contas públicas. O Ministério da Saúde (MS), com o propósito de reduzir a mortalidade associada às DCNT, assumiu o compromisso de executar ações em parceria com estados, municípios e sociedades brasileiras de cardiologia, hipertensão, nefrologia e diabetes, além de federações nacionais de portadores dessas doenças e Conselhos Nacionais dos Secretários Estaduais e Municipais de Saúde, para apoiar a reorganização da rede de saúde, com melhoria da atenção aos portadores dessas doenças. Nesse contexto, o Ministério da Saúde estabeleceu critérios e normas para a Assistência Farmacêutica aos portadores de diabetes, visando garantir o acesso aos medicamentos e aos insumos de automonitorização da glicemia (41).

Embora haja planos e iniciativas para a melhora do acesso ao medicamento e a programas assistencialistas, a realidade do paciente diabético requer sua adaptação frente ao diagnóstico, o que impõe a criação gradativa de identidade relacionada com a doença. Porém, tendo em vista a relação implícita do processo saúde-doença-cuidado com a forma de como as pessoas percebem a realidade concreta, a construção de tal identidade parece ser dificultada pela própria característica silenciosa dos primeiros sintomas, que é condição na qual o portador não se sente doente (42) e, dessa forma, torna-se essencial o cuidado farmacêutico no acompanhamento desses pacientes.

Diabetes tipo 2 e o cuidado farmacêutico. A prática do cuidado farmacêutico torna-se fundamental para promover melhor controle do diabetes tipo 2, considerando que o indivíduo adquire maior conhecimento do tratamento além de melhora na comunicação do paciente com o profissional da saúde, e de informações essen- ciais para a adesão ao tratamento. Com isso, é esperada a redução de erros de medicamentos e reações adversas, além de mudanças de hábitos fundamentais para o controle glicêmico (43).

Para avaliar o impacto da educação em saúde, um estudo foi desenvolvido para comparar os valores de $\mathrm{HbA1c}$ antes do início da atividade de educação e após seis meses, em pacientes ambulatoriais com diabetes tipo 2. Em pacientes cujos valores de $\mathrm{HbAlc} \geq 8 \%$, o nível de $\mathrm{HbAlc}$ diminuiu significativamente durante seis meses de educação ao paciente evidenciando a importância desta ação (44).

Segundo Albuquerque e cols (2015), a educação terapêutica é a ferramenta para a promoção do autocuidado em diabetes tipo 2 e deveria ser uma atividade planejada para criar condições para que pudessem produzir mudanças nos hábitos de vida (41). O uso de práticas educativas como estratégia no tratamento de diabetes tipo 2 leva à melhora no conhecimento sobre a doença $\mathrm{e}$ o tratamento, além de motivar e reforçar a importância do estilo de vida, melhorando a qualidade de vida dos pacientes por meio do aumento de sua autonomia, adesão e, assim, capacitar no manejo da enfermidade. Pacientes com esse conhecimento conseguirão reconhecer a importância da adesão à farmacoterapia e o controle deste agravo à saúde (45).

Um estudo randomizado com pacientes com diabetes tipo 2 atendidos no Universiti Kebangsaan Malaysia Medical Centre, inserido no programa Patient Education by Pharmacist Programme, foi conduzido para a avaliar a intervenção do farmacêutico em pacientes com diabetes tipo 2 em relação a vários aspectos, principalmente, adesão à farmacoterapia e qualidade de vida, após seis meses de intervenção. Os resultados mostraram a redução no nível de HbAlc e a melhora na adesão; entretanto, a melhora da qualidade de vida não teve alteração significativa entre os grupos controle e intervenção (46).

O estabelecimento de programa de consulta farmacêutica visando melhorar o tratamento de pacientes com diabetes tipo 2, e a redução de fatores de risco das complicações decorrentes, além de poder ser aplicado a outras doenças crônicas, foi desenvolvido por Zubioli e cols (2013). O programa avaliou, a cada quadrimestre durante um ano, o estilo de vida, a famacoterapia e o monitoramento por meio de exames laboratoriais, sinais vitais e antropometria. Nas consultas foi oferecida educação focada no estilo de vida e uso de medicamentos. Os autores concluíram que houve redução nos valores de glicemia, hemoglobina glicada, colesterolemia, triacilgliceridemia e pressão arterial (43). Segundo os autores, o aumento da concordância e a correção dos PRM contribuíram para a melhora do tratamento além de reduzir fatores de risco de complicações diabéticas (47).

Em estudo de metanálise com a utilização de 14 ensaios e com $n=2073$, foi avaliado o efeito da interven- 
ção farmacêutica no controle glicêmico em população diabética. Os resultados dmostraram associações estatisticamente e clinicamente significativas entre a intervenção do farmacêutico e a melhora no controle glicêmico (48).

Dentre os estudos citados destaca-se o publicado por Obreli-Neto e cols (2011), mostrando que os custos da assistência farmacêutica não representaram significativamente o aumento do custo total direto (49). Além disso, em um estudo mais crítico de custo realizado por Picoli (2015), além de maior efetividade para os parâmetros de controle glicêmico, HbAlc e aumento de ganho vida comparado ao grupo sem a prática do cuidado farmacêutico, o benefício financeiro levantado pelos gestores da saúde foi conclusivo e serviu de suporte para as tomadas de decisão para políticas de saúde relacionadas ao uso racional de recursos, além da valorização e da importância do profissional farmacêutico para a promoção de saúde e da qualidade de vida aos pacientes (40).

Para avaliar a efetividade dos cuidados farmacêuticos no controle de parâmetros clínicos (glicemia de jejum e hemoglobina glicosilada), um estudo retrospectivo realizado no Centro de Formação e Saúde Comunitária da Faculdade de Medicina de Ribeirão Preto, SP, envolveu pacientes com diabetes tipo 2 monitorados por doze meses. Os autores concluíram que os valores médios de glicemia de jejum no grupo de intervenção foram significativamente reduzidos, o que foi verificado, também com os níveis de hemoglobina glicosilada. Acrescido aos benefícios decorrentes dos cuidados farmacêuticos houve a resolução de quase $50 \%$ dos problemas de terapia medicamentosa identificados. Portanto, a informação fornecida pelo farmacêutico a portadores de diabetes tipo 2 aumentou a adesão ao tratamento e o controle glicêmico (50).

De acordo com a análise de Pepe e cols (2010), foi possível observar que os resultados obtidos por meio de cuidados farmacêuticos vêm permitindo aos gestores conhecer, de forma sistematizada, os indicadores para viabilizar o correto uso dos medicamentos financiados pelo SUS. Nessa perspectiva, o cuidado farmacêutico funciona como marco importante para o trabalho dos Comitês de Uso Racional de Medicamentos, no planejamento e em ações que possam minimizar problemas sistêmicos na prescrição e no decorrer do tratamento do paciente (51).

Embora o cenário do cuidado farmacêutico venha ganhando robustez no Brasil, essa prática ainda enfrenta dificuldades para sua implantação. $\mathrm{O}$ acesso a medicamento por parte dos usuários do SUS é atualmente um grande desafio a ser superado pelos gestores públicos nas Unidades Básicas de Saúde. A ausência de profissionais farmacêuticos e, ainda, a escassez de documentação científica que possibilite demonstrar, aos gestores do sistema público e privado, que a prática do cuidado farmacêutico representa investimento e não custo, vem sendo discutida há anos (52). Vale ressaltar que, no Brasil, o farmacêutico, na maioria das vezes, acumula funções administrativas, prejudicando, assim o tempo disponível para atender os pacientes de forma completa, que seria não apenas a orientação de balcão, mas também a prática de serviços clínicos de sua competência. O medicamento é importante para a melhora da saúde, entretanto a sua efetividade depende, dentre outros fatores, do seu uso racional, cuja informação deverá estar baseada em evidência científica de maneira independente e imparcial.

A prática do cuidado farmacêutico requer mudança estrutural das farmácias em todos nichos e, também, um rearranjo de funções. Algumas das dificuldades para sua implantação têm sido observadas em vários países e refletem a crise de identidade profissional e, em consequência, a falta de reconhecimento social e baixa inserção na equipe multiprofissional de saúde. Além disso, as farmácias públicas não têm inserção no sistema de saúde. O conhecimento da prática do cuidado farmacêutico mostrou-se limitado no âmbito desses profissionais, situação que pode vir a alterar-se, à medida que as mudanças curriculares em curso surtam efeito na formação dos novos farmacêuticos (53).

É necessário salientar, entretanto, que muito lentamente, ocorre uma reação positiva à intensificação das atividades clínicas do farmacêutico em serviços públicos. A Secretaria Municipal de Saúde de São Paulo (SMS-SP) publicou, recentemente, a Portaria SMS.G n. 1.918 (em 27 de outubro de 2016) que "Institui os Cuidados Farmacêuticos no âmbito da SMS-SP", definindo diretrizes para organização do serviço farmacêutico, conciliando as atividades clínicas e as administrativas para promover o desenvolvimento de ações voltadas ao Cuidado Farmacêutico nas unidades da rede básica e de especialidades da SMS-SP. Apresenta como objetivos, melhorar os resultados terapêuticos individuais e coletivos na gestão do cuidado em saúde por meio de ações clínicas do farmacêutico integradas à equipe multiprofissional; promover o uso racional de medicamentos junto aos prescritores, equipes de saúde e a comunidade, englobando ações de educação em saúde e farmacovigilância (54). Portanto, em âmbito municipal, a sensibilização já resultou em ação de impacto nas Unidades Básicas de Saúde e, talvez, esta ação possa ser multiplicada e desdobrada.

Em 2011, o Ministério da Saúde lançou seu Plano de Ações Estratégicas para o Enfrentamento das Doenças Crônicas Não Transmissíveis, enfatizando ações populacionais para controlar as doenças cardiovasculares, o diabetes, o câncer e a doença respiratória crônica, predominantemente pelo controle do fumo, inatividade física, alimentação inadequada e uso prejudicial de álcool (20). Neste contexto, o Estudo Longitudinal de Saúde Adulto (ELSA Adulto), desde 2008, acompanha 15.105 servidores públicos do País e seus dados espelham a realidade brasileira de altas prevalências de diabetes e 
hipertensão e dos fatores de risco. Com isso, a diversidade das informações produzidas permitirá aprofundar o entendimento causal dessas doenças e subsidiar políticas públicas para o seu enfrentamento (18).

\section{CONCLUSÃO}

Existem diversos fatores que poderão ser contornados com a simples orientação farmacêutica que, infelizmente, é deficiente em muitas Unidades de Saúde Pública do SUS; e o cuidado farmacêutico precisa estar presente de maneira organizada e efetiva para o sucesso e a adesão terapêutica pois envolve o acompanhamento e o monitoramento farmacoterapêuticos próximos ao indivíduo, não apenas em relação ao uso farmacológico, mas também, de mudanças de hábitos e estilo de vida que são essenciais para a promoção de Saúde e a racionalização do uso de medicamentos.

Em relação aos benefícios conseguidos e, no que tange $\mathrm{o}$ aspecto econômico desta prática, torna-se concreto e mensurável o benefício e a viabilidade da extensão do cuidado farmacêutico devendo, portanto, ser contemplado de maneira ampla, nos recursos disponibilizados para o investimento e a promoção de Saúde no SUS.

\section{REFERÊNCIAS}

1. BRASIL. Ministério da Saúde. Secretaria de Ciência, Tecnologia e Insumos Estratégicos. Departamento de Assistência Farmacêutica e Insumos Estratégicos. Cuidado farmacêutico na atenção básica. Caderno 1. Serviços farmacêuticos na atenção básica à saúde. $1^{\mathrm{a}}$ ed. rev. Brasília, DF: Ministério da Saúde, 2015.

2. PCNE Pharmaceutical Care Network Europe. Disponível em: http://www.pcne.org/working-groups Acesso em 21 ago 2016.

3. WHO. World Health Organization. Declaration on occupational health for all. Geneva. 1994.

4. Bernsten C, Björkman I, Caramona M, Crealey G, Frøkjaer B, Grundberger E, Gustafsson T, Henman M, Herborg H, Hughes C, McElnay J, Magner M, van Mil F, Schaeffer M, Silva S, Søndergaard B, Sturgess I, Tromp D, Vivero L, Winterstein A. Improving the well-being of elderly patients via community pharmacy-based provision of pharmaceutical care. Drugs Aging. 2001;18(1):63-77.

5. Brandão A. FIP: Vocação da Farmácia é orientar paciente. Braz J Pharm Sci. 1996;3:27.

6. WHO. World Health Organization. The role of the pharmacist in the health care system: Preparing the future pharmacist. Geneva. 1997.

7. EDQM. European Directorate for the Quality of Medicines \& HealthCare, Pharmaceutical Care. Policies and Practices for a Safer, More Responsible and Cost-effective Health System. Strasbourg: Directorate for the Quality of Medicines \& HealthCare of the Council of Europe (EDQM). 2012. Disponível em: https:// www.edqm.eu/medias/fichiers/policies_and_practices_for_a_safer_more_responsibl.pdf. Acesso em 14 set $201 \overline{6}$.

8. Vale EG, Pagliuca LMF. Construção de um conceito de cuidado de enfermagem: contribuição para o ensino de graduação. Rev Bras Enferm. 2011;64(1). DOI: 10.1590/ S0034-71672011000100016
9. CFF. Conselho Federal de Farmácia. Serviços farmacêuticos diretamente destinados ao paciente, à família e à comunidade: contextualização e arcabouço conceitual. Brasília: Conselho Federal de Farmácia; 2016.

10. Hepler CD, Strand LM. Opportunities and responsibilities in pharmaceutical care. Am J Health-Syst Pharm. 1990;47(3):533-543.

11. CFF. Conselho Federal de Farmácia. Resolução n. 585, de 29 de agosto de 2013. Regulamenta as atribuições clínicas do farmacêutico e dá outras providências. Disponível em: http://www.cff.org.br/userfiles/file/resolucoes/585.pdf Acesso em 14 set 2016.

12. BRASIL. Presidência da República. Casa Civil. Subchefia para Assuntos Jurídicos. Lei 13.021, de 08 de agosto de 2014. Dispõe sobre o exercício e a fiscalização das atividades farmacêuticas. Disponível em: http://www. planalto.gov.br/ccivil_03/_Ato2011-2014/2014/Lei/ L13021.htm Acesso em 21 ago 2016.

13. Correa-de-Araújo, R. O papel do farmacêutico na prevenção de problemas relacionados ao uso de medicamentos. Resultados do projeto Fleetwood. Farmacoterapêutica. 2001;6(2):63-66.

14. Strand LM, Cipolle RJ, Morley PC, Frakes MJ. The impact of pharmaceutical care practice on the practitioner and the patient in the ambulatory practice setting: twenty-five years of experience. Curr Pharm Des. 2004;10:3987-4001.

15. Lira-Júnior DP. Impacto de um programa de Atenção Farmacêutica, o cuidado de um grupo de idosos atendidos na Unidade Básica Distrital de Saúde Dr. Ítalo Baruffi, Ribeirão Preto (SP). 2005. 156 f. Tese (Doutorado em Ciências Farmacêuticas) - Universidade de São Paulo, São Paulo, 2005.

16. Silva JVF, Silva EC, Rodrigues PARA, Miyazawa AP. A relação entre o envelhecimento populacional e as doenças crônicas não transmissíveis: Sério desafio de saúde pública. CGBS. 2015;2(3):91-100. 
17. BRASIL. Portal da Saúde. 2016. Disponível em: http:// portalsaude.saude.gov.br/ Acesso em 21 ago 2016.

18. Duncan BB, Chor D, Aquino EMI, Bensenor IM, Mill JG, Schmidt MI, Lotufo PA, Vigo A, Barreto SM. Doenças crônicas não transmissíveis no Brasil: Prioridade para enfrentamento e investigação. Rev Saúde Públ. 2012;46(supp):126-134. DOI: 10.1590/ S0034-89102012000700017

19. BRASIL. Ministério da Saúde. Secretaria de Vigilância em Saúde. Departamento de Análise de Situação de Saúde. Plano de ações estratégicas para o enfrentamento das Doenças Crônicas Não Transmissíveis (DCNT) no Brasil 2011-2022. Brasília: Ministério da Saúde; 2011.

20. Malta DC, Stopa SR, Szwarcwald CL, Gomes NL, Silva-Júnior JB, Chioro-dos-Reis AA. A vigilância e o monitoramento das principais doenças crônicas não transmissíveis no Brasil - Pesquisa Nacional de Saúde, 2013. Rev Bras Epidemiol. 2015;18(2)3-16. DOI: 10.1590/19805497201500060002

21. DATASUS. Departamento de Informática do SUS. Disponível em: http://datasus.saude.gov.br/ Acesso em 30 jun 2016.

22. Nobre F. Sociedade Brasileira de Cardiologia. VI Diretrizes Brasileiras de Hipertensão. 2010. Arq Bras Cardiol. 2016. Disponível em: http://publicacoes.cardiol.br/consenso/2010/Diretriz_hipertensao_associados.pdf Acesso em 14 set 2016.

23. Santa-Helena ETS, Nemes MIB, Eluf-Neto J. Desenvolvimento e validação de questionário multidimensional para medir não-adesão ao tratamento com medicamentos. Rev Saúde Públ. 2008;42(4);764-767. DOI: 10.1590/ s0034-89102008000400025

24. WHO. World Health Organization. A global briefing on hypertension: silent killer, global public health crisis. Geneva. 2013. Disponível em: http://www.who.int/cardiovascular_diseases/publications/global_brief_hypertension/en/ Acesso em 10 ago 2016.

25. Lessa I. Impacto social da não-adesão ao tratamento da hipertensão arterial. Rev Bras Hipertens. 2006;13(1):3946.

26. Cazarim MS, Freitas O, Penaforte TR, Achcar A, Pereira LRL. Impact assessment of Pharmaceutical Care in the management of hypertension and coronary risk factors after discharge. Plos One. 2016;11(6):1-14. DOI: 10.1371/journal.pone.0155204

27. Robinson JD, Segal R, Lopez LM, Doty RE. Impact of a Pharmaceutical Care intervention on blood pressure control in a chain pharmacy practice. AOP. 2009;44(1):8896. DOI: 10.1345/aph.11289

28. Martins BPR, Aquino AT, Provin MP, Lima DM, Dewulf NLS, Amaral RG. Pharmaceutical Care for hypertensive patients provided within the Family Health Strategy in Goiânia, Goiás, Brazil. Braz J Pharm Sci. 2013;49(3):609618. DOI: $10.1590 / \mathrm{s} 1984-82502013000300023$
29. Oliveira EM, Spiri WC. Programa Saúde da Família: a experiência de equipe multiprofissional. Rev Saúde Públ. 2006;40(4):727-733. DOI: 10.1590/s003489102006000500025

30. Rita OA, Rukeme J. Ogigirigi. Patients' perception of medication therapy management in a hospital pharmacy setting.UK J Pharm Biosci.2016;4(2):24-30. DOI: 10.20510/ukjpb/4/i2/97085

31. Lyra-Júnior DP, Marcellini OS, Pelá IR. Effect of pharmaceutical care intervention on blood pressure of elderly outpatients with hypertension. Rev Bras Ciênc Farm. 2008;44(3):451-457. DOI: 10.1590/S151693322008000300015

32. Souza TT, Godoy RR, Rotta I, Pontarolo R, Fernandez-Llimos F, Correr CJ. Morbidade e mortalidade relacionadas a medicamentos no Brasil: revisão sistemática de estudos observacionais. Rev Ciênc Farm Básica Apl. 2013;4(35):519-532.

33. Milech A et al. Sociedade Brasileira de Diabetes. Diretrizes da Sociedade Brasileira de Diabetes (2015-2016). 2016. Disponível em: http://www.diabetes.org.br/sbdonline/images/docs/DIRETRIZES-SBD-2015-2016.pdf Acesso em 20 ago 2016

34. WHO. World Health Organization. Global reports on diabetes. 2016. Disponível em: http://www.who.int/diabetes/global-report/en/ Acesso em 10 ago 2016.

35. Gallagher J. Diabetes quadruplica e já mata 1,5 milhão de pessoas ao ano, alerta OMS. BBC Brasil. Postado em 06 abr 2016. Disponivel em: http://www.bbc.com/portuguese/noticias/2016/04/160406_diabetes_aumenta_lab Acesso em 01 jul 2016.

36. BRASIL. Ministério da Saúde. Secretaria de Atenção à Saúde. Departamento de Atenção Básica. Diabetes mellitus. Brasília: Ministério da Saúde; 2006.

37. Seuring T, Archangelidi O, Suhrcke M. The economic costs of type 2 diabetes: A global systematic review. Pharmacoeconomics. 2015;33(8):811-831. DOI: 10.1007/s40273-015-0268-9

38. IDF. International Diabetes Federation. Diabetes Atlas. 7th Edition. 2016. Disponível em: http://www.diabetesatlas.org/disclaimer.html Acesso em 15 set 2016.

39. ADA. American Diabetes Association. Economic costs of diabetes in the U.S. in 2012. Diabetes Care. 2013;36(4):1033-1046. DOI: 10.2337/dc12-2625

40. Picoli RM. Análise de custo efetividade da atenção farmacêutica no tratamento de diabetes mellitus tipo 2 . 2015. 148f. Defesa de mestrado. Faculdade de Economia, Administração e Contabilidade de Ribeirão Preto, Universidade de São Paulo, Ribeirão Preto, 2015.

41. BRASIL. Portal Brasil. Saúde. Remédio gratuito. Programa do Governo Saúde Não Tem Preço. Disponível em: https://www.brasil.gov.br/saude/2012/04/remedio-gratuito Acesso em 14 set 2016. 
42. Olshansky E, Sacco D, Fitzgerald K, Zickmund S, Hess R, Bryce C, McTigue K, Fischer G. Living with diabetes: Normalizing the process of managing diabetes. Diabetes Educ. 2008;34(6):1004-1012. DOI: $10.1177 / 0145721708327304$

43. Pereira LRL, Freitas O. A evolução da Atenção Farmacêutica e a perspectiva para o Brasil. Rev Bras Ciênc Farm. 2008;44(4):601-612. DOI: 10.1590/s151693322008000400006

44. Ichiki Y, Kobayashi D, Kubota T, Ozono S, Murakami A, Yamakawa Y, Zeki K, Shimazoe T. Effect of patient education for diabetic outpatients by a hospital pharmacist: a retrospective study. Yakugaku Zasshi. 2016;136(12):166716674. DOI:10.1248/yakushi.16-00081

45. Albuquerque $\mathrm{C}$, Correia $\mathrm{C}$, Ferreira $\mathrm{M}$. Adherence to the therapeutic regime in person with type 2 Diabetes. Procedia - Soc Behav Sci. 2015; 17:350-358. DOI: 10.1016/j. sbspro.2015.01.132

46. Butt M, Ali AM, Bakry MM, Mustafa N. Impact of a pharmacist led diabetes mellitus intervention on $\mathrm{HbA1c}$, medication adherence and quality of life: A randomised controlled study. Saudi Pharm J. 2016;24(1):40-48. DOI: 10.1016/j.jsps.2015.02.023

47. Zubioli A, Silva MARCP, Tasca RS, Curi R, Bazotte RB. Pharmaceutical consultation as a tool to improve health outcomes for patients with type 2 diabetes. Braz J Pharm Sci. 2013;49(1):85-94. DOI: 10.1590/s198482502013000100010

48. Collins C, Limone BL, Scholle JM, Coleman CI. Effect of pharmacist intervention on glycemic control in diabetes. Diabetes Res Clin Pract. 2011;92(2):145-152. DOI: 10.1016/j.diabres.2010.09.023
49. Obreli-Neto PR, Guidoni CM, Baldoni AO, Pilger D, Cruciol-Souza JM, Gaeti-Franco WP, Cuman RKN. Effect of a 36-month pharmaceutical care program on pharmacotherapy adherence in elderly diabetic and hypertensive patients. Int J Clin Pharm. 2011;33(4):642-649. DOI: $10.1007 / \mathrm{s} 11096-011-9518-\mathrm{x}$

50. Borges APS, Borges AP, Guidoni CM, Ferreira LD, de Freitas O, Pereira LR. The pharmaceutical care of patients with type 2 diabetes mellitus. Pharm World Sci. 2010;32(6):730-736. DOI 10.1007/s11096-010-9428-3

51. Pepe VLE, Figueiredo TA, Simas L, Osório-de-Castro CGS, Ventura M. A judicialização da saúde e os novos desafios da gestão da assistência farmacêutica. Ciênc Saúde Col. 2010;15(5):2405-2414. DOI: 10.1590/s141381232010000500015

52. Boing $\mathrm{AC}$, Boing $\mathrm{AF}$. Hipertensão arterial sistêmica: o que nos dizem os sistemas brasileiros de cadastramentos e informações em saúde. Rev Bras Hipertens. 2007;14(2):84-88. Disponível em: http://departamentos. cardiol.br/dha/revista/14-2/06-hipertensao.pdf Acesso em 30 jun 2016.

53. Farina SS, Romano-Lieber NS. Atenção farmacêutica em farmácias e drogarias: Existe um processo de mudança? Saúde Soc. 2009;18(1):7-18. DOI: 10.1590/S010412902009000100002

54. SMS-SP. Secretaria Municipal de Saúde de São Paulo. Portaria SMS n. 1918/16 de 27 de outubro de 2016 - Institui os Cuidados Farmacêuticos em SMS. 2016. Disponível em: http://www3.prefeitura.sp.gov.br/cadlem/ secretarias/negocios juridicos/cadlem/integra.asp?al$\mathrm{t}=27102016 \mathrm{P} \% 20019182016 \mathrm{SMS}$ Acesso em $12 \mathrm{dez}$ 2016. 